\title{
Persistent Mullerian Duct Syndrome Type ।
}

National Cancer Institute

\section{Source}

National Cancer Institute. Persistent Mullerian Duct Syndrome Type I. NCI Thesaurus.

Code C120189.

Persistent Mullerian duct syndrome due to deficiency of anti-Mullerian hormone. 